\title{
A highly configurable and efficient simulator for job schedulers on supercomputers
}

April 12, 2013 | Carsten Karbach, Jülich Supercomputing Centre (JSC) 


\section{Motivation}

\section{Objective}

- Simulation of supercomputer job schedulers for prediction of job start times

- Features of simulation program:

- Efficient and configurable

- Extensible and generic

- Use cases:

- User $\rightarrow$ predicts start time of his own jobs

- Administrator $\rightarrow$ configurable simulation of supercomputer, throughput optimization

- Problem: unpublished scheduling algorithms, job schedulers do not provide global on-line prediction 


\section{Motivation}

\section{Solution}

- JuFo (Jülich Forecast):

$\mathrm{C}++$ application using data format $\mathbf{L M L}$

- Based on prediction component of LLview

- LLview provides status information of supercomputers in LML

- Abstraction of scheduling systems Moab and Loadleveler 


\section{Part I: Problem definition}

April 12, 2013 | Carsten Karbach, Jülich Supercomputing Centre (JSC) 


\section{Problem definition}

\section{What is the objective of JuFo?}

- On-line simulation of global job schedulers

- LML as interface to LLview, input and output of JuFo

- Prediction of job dispatch time and used resources

\section{global job scheduler}

- Prioritizes and places jobs

- Fixed CPU set is allocated to each job

- Handling of reservations, queues and dependencies

- Examples: Moab (JUROPA), Loadleveler (JUQUEEN) 


\section{The scheduling problem}

- Supercomputer offers resources e.g. CPUs, GPUs, memory

- Jobs request resources, wait in queues until resources become available

- Problem: generate optimal schedule for all waiting jobs

- Optimization over time and resources

- Approximation with FCFS, List-Scheduling and Backfilling

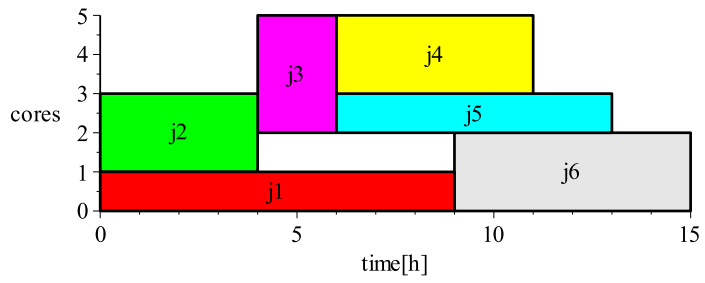




\section{Part II: Embedding JuFo}

April 12, 2013 | Carsten Karbach, Jülich Supercomputing Centre (JSC) 


\section{Embedding JuFo}

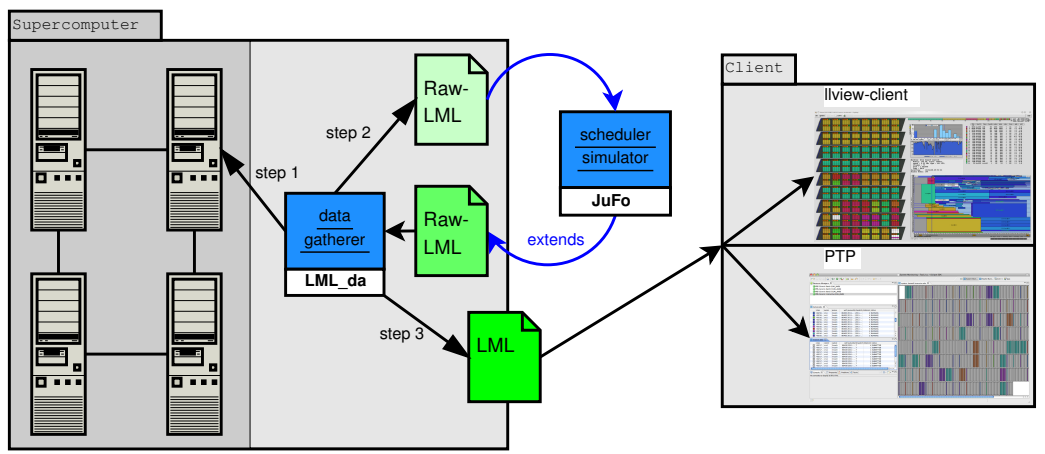




\section{Data format}

\section{Large-scale system Markup Language (LML)}

- Data format for status information of supercomputers

- Based on XML, specified by an XML Schema

- Interface for LML_da, JuFo and Clients

- LLview client visualizes JuFo's outputs

\section{Input data required by JuFo}

- Compute nodes: number of processors per node

- Queues: grouping of jobs, scheduling rules

- Jobs: running and waiting; requested resources 


\section{Part III: Scheduling algorithms}

April 12, 2013 | Carsten Karbach, Jülich Supercomputing Centre (JSC) 


\section{Constraints}

- No job migration

- No preemption

- Wall clock limit (WCL) is mandatory for each job

- WCL is used as actual job run-time in JuFo 


\section{Scheduling Algorithms}

- FCFS: Job with longest queue time starts first

- List-Scheduling: Prioritization by arbitrary formula, lower ranked jobs can run before higher ranked jobs

- Backfilling: Reservations for top dogs, fill idling resources with smaller jobs

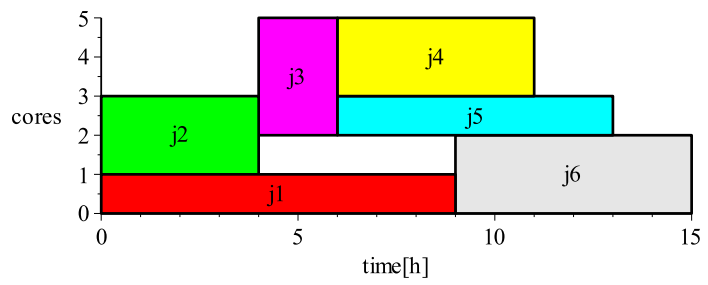




\section{Further requirements}

- Prioritization JUROPA:

systemprio + userprio $+10 *$ nodes + qtime + qtime/wall $-300 *$ actjobs

- Job dependencies

- Reservations

- Resource requests: nodes, CPUs, GPUs, global/per node, network topology

- Nodesharing

- Top dogs per queue

- Queue defines scheduling constraints:

- Allowed nodes

- Limits number of active/waiting jobs 


\section{Part IV: Design of JuFo}

April 12, 2013 | Carsten Karbach, Jülich Supercomputing Centre (JSC) 


\section{Package overview}

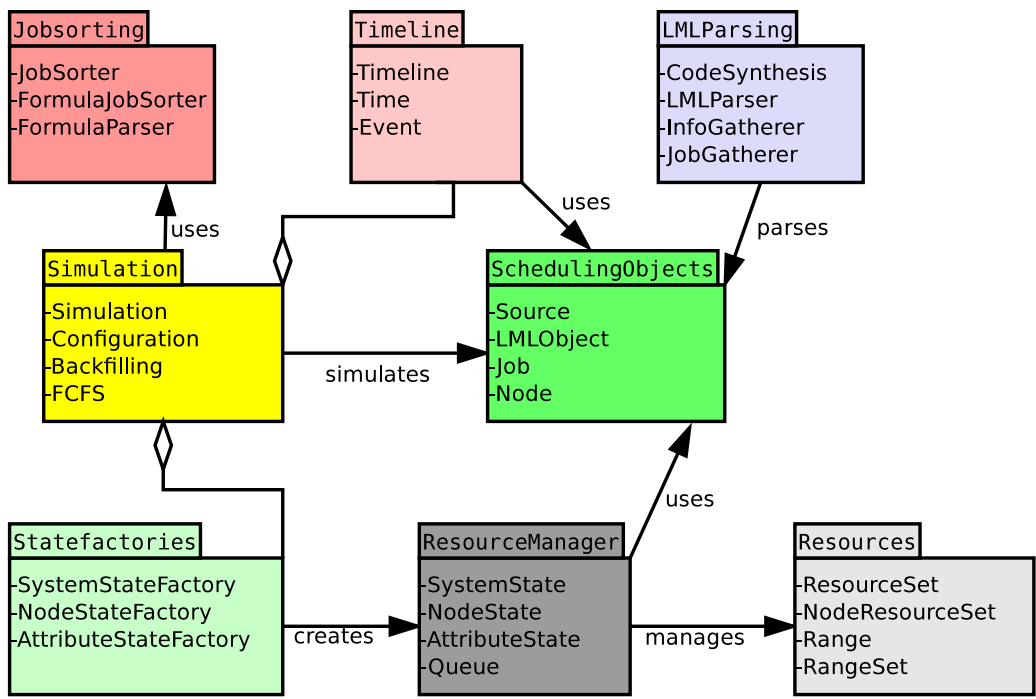




\section{Target}

- Packages are encapsulated

- Interaction only via interfaces, actual implementation unknown to foreign packages

- Reference implementation for each interface

\section{Result}

- Extensible basis for job scheduler simulation

- Well defined extension points for new sorting/scheduling algorithms and resource managers

- Arbitrary combination of available sorting/scheduling algorithms and resource managers 


\section{Part V: Validation}

April 12, 2013 | Carsten Karbach, Jülich Supercomputing Centre (JSC) 


\section{How to validate results?}

1 Log all events for a given time span (new jobs, early job completion, canceling waiting jobs)

2 Run JuFo for this time span using exact WCLs

3 Compare reality with prediction 


\section{Validation results for JUROPA}

- Validation framework

- JuFo is best configured for JUROPA

- Validation run on 8 different days

\section{Results}

- Time span: 2 - 5 hours

- Jobs: 100-280 per day

- Ø Error: 1-13 minutes

- High variance due to missing information

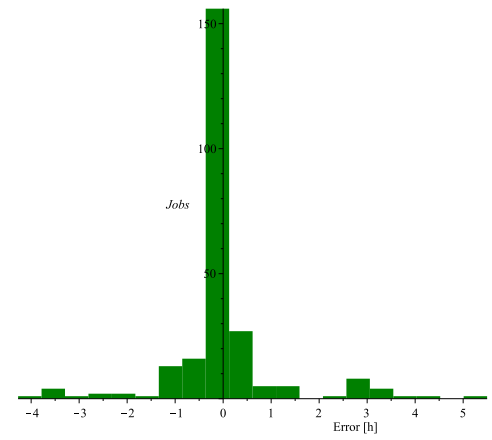




\section{Part VI: Outlook}

April 12, 2013 | Carsten Karbach, Jülich Supercomputing Centre (JSC) 


\section{Outlook}

- Configuration and validation for JUQUEEN, JUDGE

- Parallelization for higher efficiency

- Visualization: in PTP, new visualization methods 


\section{Thank you for your attention!}




\section{Summary}

- JuFo: extensible simulation for global job schedulers

- LML as data format

- LML_da collects input data

- Scheduling algorithms: FCFS, List-Scheduling, Backfilling

- Analysis of Loadleveler and Moab

$\rightarrow$ Simulation design

- Validation framework, successful on JUROPA 


\section{Backfilling implementation}

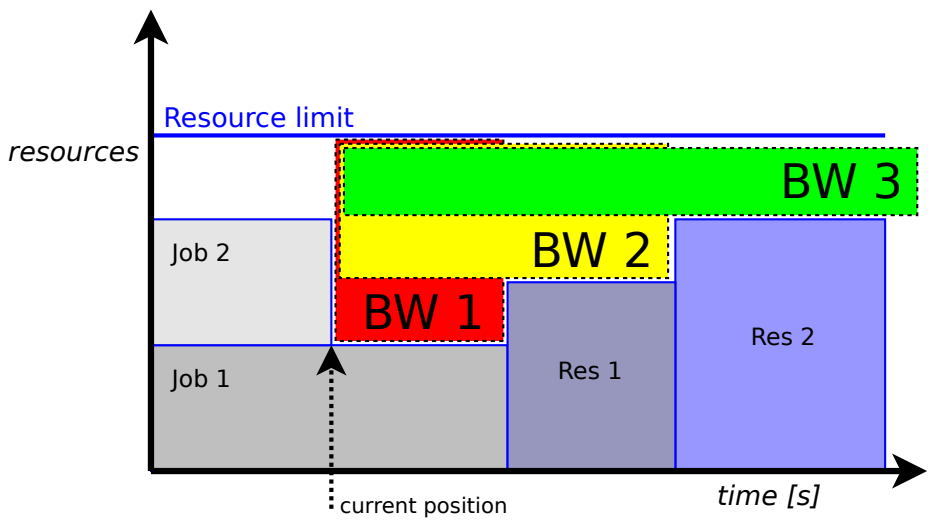

- Backfill window: resources $\times$ time span 


\section{Scheduling problem - mathematical definition}

- Schedule $s=\left(s_{1}, \ldots, s_{n}\right)^{T}$ with $s_{j}$ as dispatch time of job $j$

- $V=\left\{s \in \mathbb{R}_{\geq 0}^{n} \mid s\right.$ is valid $\}$, all allowed schedules

- $f$ : objective function, e.g. $f(s):=-\max _{j \in J}\left(s_{j}+w_{j}\right)$, $w_{j}$ WCL j

- find $s_{o p t}$ with $f\left(s_{o p t}\right)=\max _{s \in V} f(s)$

- Derived problem from resource-constrained project scheduling problem (RCPSP)

- RCPSP is NP-hard

$\rightarrow$ no solution in polynomial time

- Instead approximation with

- First-Come-First-Served (FCFS)

- List-Scheduling

- Backfilling 


\section{Implementation FCFS}

void fffs(waitingJobs)

\begin{tabular}{|c|c|}
\hline \multicolumn{2}{|l|}{ timePos $=0$} \\
\hline \multicolumn{2}{|c|}{ timePos $<$ timeline.size () \& \& waitingJobs.size ()$>0$} \\
\hline \multicolumn{2}{|l|}{ react on event at timePos } \\
\hline \multicolumn{2}{|l|}{ waitingJobs.size ()$>0$} \\
\hline \multicolumn{2}{|c|}{ is waitingJobs[0] insertable } \\
\hline insert job into system at timePos & \\
\hline waitingJobs.erase( waitingJobs[0] ) & break \\
\hline
\end{tabular}




\section{Summary of JuFo packages}

- Simulation: implements scheduling algorithms

- ResourceManager: manages compute resources; decides, whether a job can be started on given resources

- Jobsorting: configurable prioritization of waiting jobs

- Timeline: stores all simulation events such as job dispatch, completion and reservations

- LMLParsing: reads input LML, converts into object hierarchy, generates output LML 


\section{Validation results JUROPA - Details I}

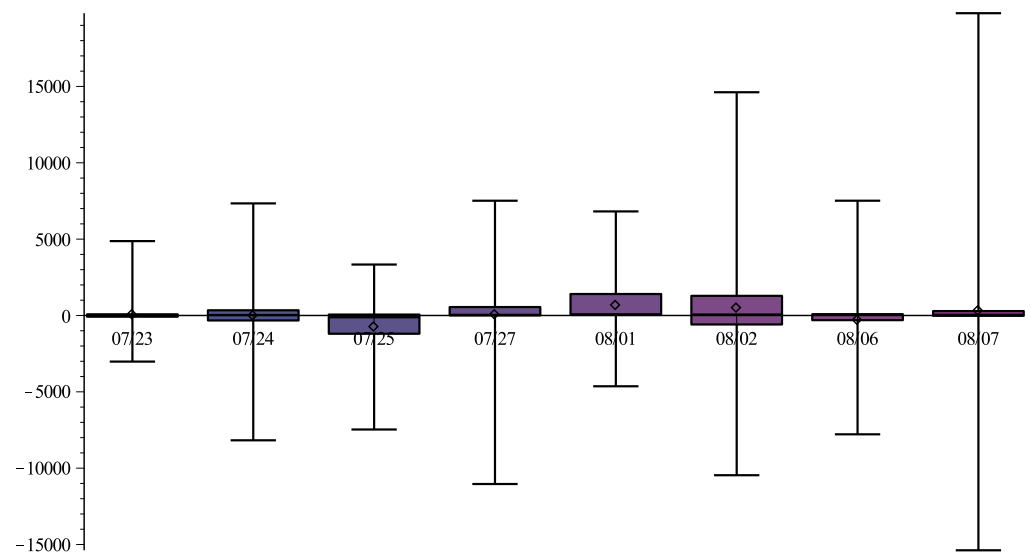




\section{Validation results JUROPA - Details II}

- Difference between users' WCL and actual job time span: on average 2-4 hours

- Accuracy of prediction based on users' wall comp. bad (average error of multiple hours)

- Target of JuFo is not exact prediction, but modeling the job scheduler based on input data provided by LML_da

- Better results expected by combining JuFo with statistical data for WCL 


\section{JuFo for JUROPA - improvements}

- Use actual contingent data for job priorities instead of showq outputs $\rightarrow$ system specific extension of LML_da

- Jobs with identical system priority are sometimes sorted wrong $\rightarrow$ improve details of job sorting

- Reservations are not collected by LML_da $\rightarrow$ extension of LML_da

- Dynamic querying of queue-configuration $\rightarrow$ extension/configuration of LML_da 


\section{JUROPA - prediction}

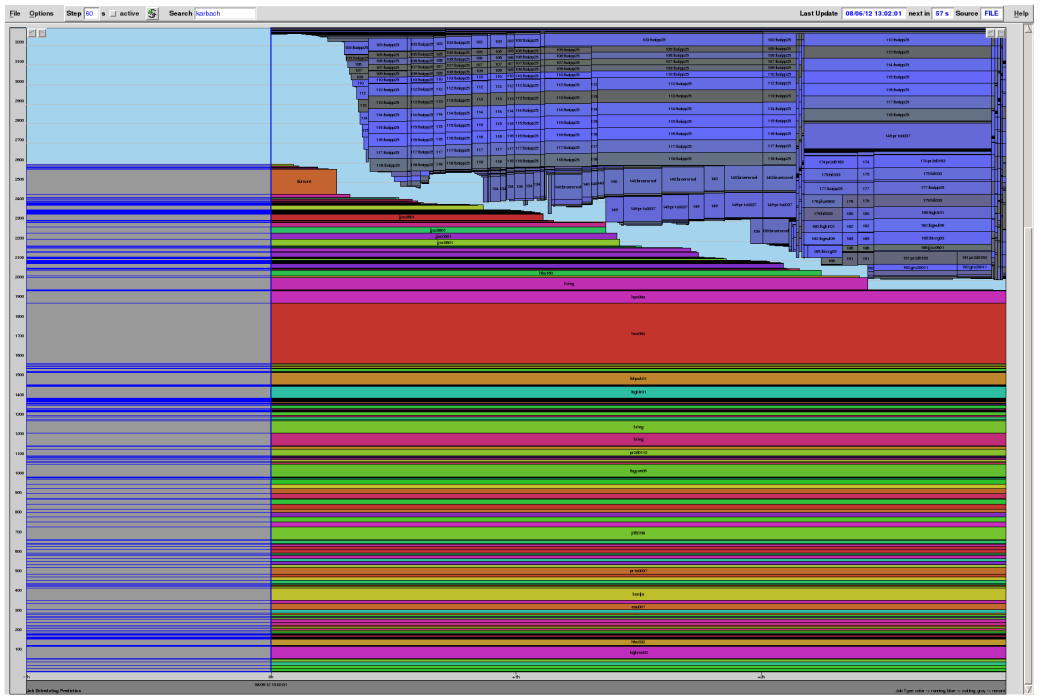




\section{JUROPA - actual schedule}

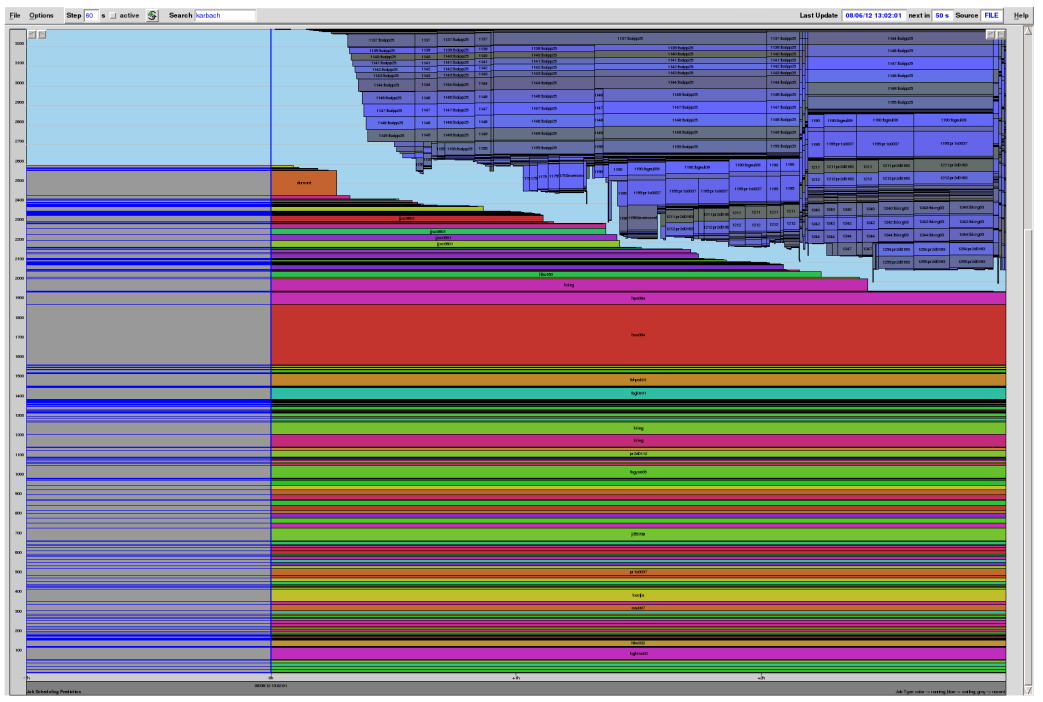




\section{Part I: LLview's prediction}

April 12, 2013 | Carsten Karbach, Jülich Supercomputing Centre (JSC) 


\section{LLview}

\section{- monitors supercomputer status}

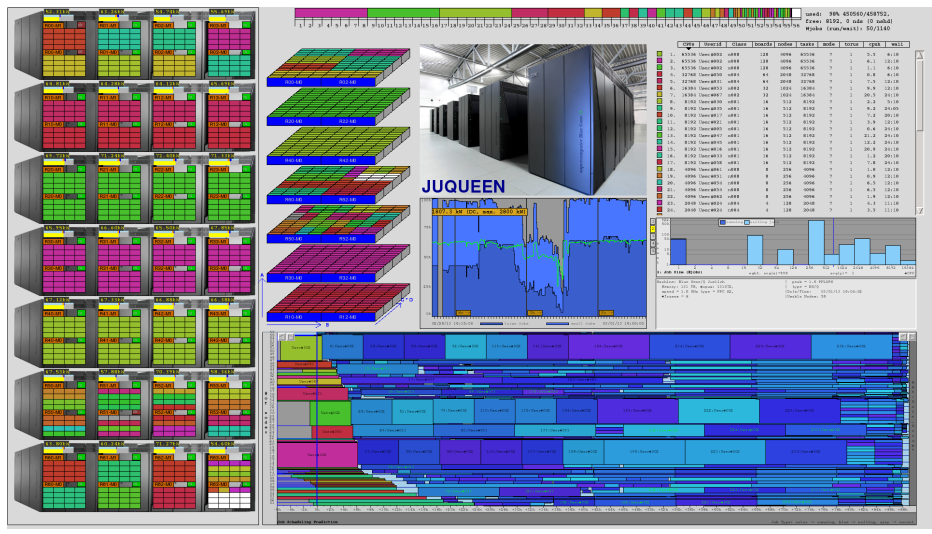

Source: Snapshot LLview for JUQUEEN (IBM BG/Q, 450k cores, 5.9 Petaflops) 


\section{LLview's prediction - SchedSim}

- SchedSim is written in Perl

- Especially designed and tested for Loadleveler systems

- Basis for design of JuFo

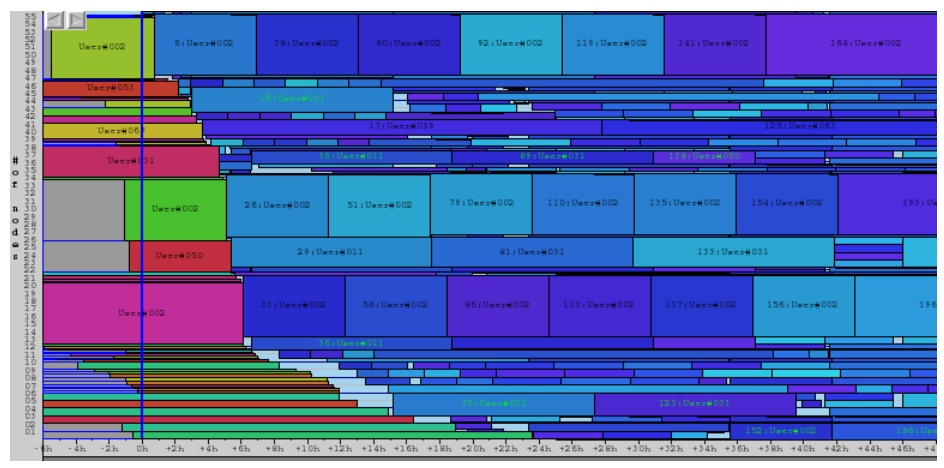

Source: Snapshot LLview for JUQUEEN 


\section{SchedSim - Analysis}

\section{Pro}

+ Highly configurable

+ Advanced modeling of JUGENE

+ Simple installation, embedded into LLview

\section{Contra}

- Limited scalability and performance

- Hard to extend

- Based on old implicit XML format, which is to be replaced by LML 


\section{Part II: Job schedulers}

April 12, 2013 | Carsten Karbach, Jülich Supercomputing Centre (JSC) 


\section{Loadleveler vs. Moab}

\section{Loadleveler}

- JUGENE, JUQUEEN

- Consists of scheduler, execution machine and central manager

- Considers torus-network

- No simulation mode, prediction only for top dogs

- Backfilling

\section{Moab}

- JUROPA, JUDGE

- Scheduler using Torque as resource manager

- Network irrelevant

- Simulation mode and showstart

- Backfilling with backfill window first 


\section{Similarities}

- Reservations

- Highly configurable

- Compute nodes offer resources, jobs request them

- Main components:

1 Job sorting

2 Scheduling algorithm

3 Resource manager 


\section{Part III: Optimization}

April 12, 2013 | Carsten Karbach, Jülich Supercomputing Centre (JSC) 


\section{Serial Optimization}

- Similar jobs: take advantage of jobs with similar requests (queue, number CPUs, WCL)

- Simultaneous events: execute events with identical time stamp in one iteration; reduces overall number of iterations

- Backfill windows: generate backfill windows and place jobs into them, instead of forward simulation separately for each job

- Recursive interval data structure: Use intervals for storing compute resources instead of expanded trees 


\section{Recursive interval data structure}

- JuFo manages compute nodes and CPUs per node

- Jobs are allocated to resources, e.g. job 1 uses nodes 10-15

- Idea 1: Tree $\rightarrow$ simple, but not efficient and memory demanding

- Idea 2: Recursive intervals $\rightarrow$ efficient, but more complex operations
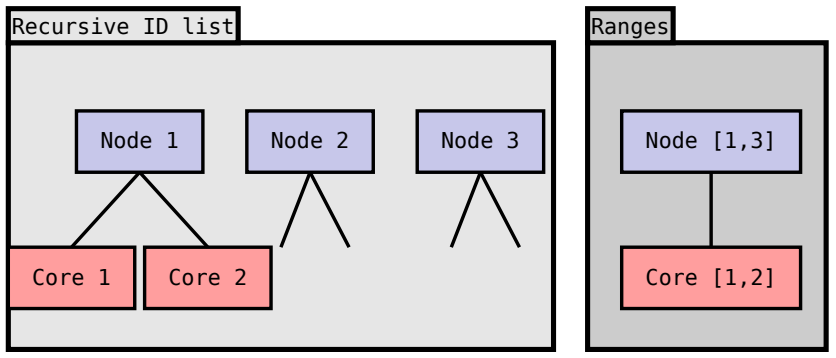


\section{Profile analysis I}

\section{Problem}

- How much simulation time is spent for each component?

\section{Solution}

- Profile analysis with gprof

- Investigate JuFo runs on JUROPA: Backfilling, 1 top dog per queue, divided into JSC and HPC-FF

- 10 input samples with 900-1600 jobs, more than 3000 nodes, simulation time $16-65 \mathrm{~s}$ 


\section{Profile analysis II}

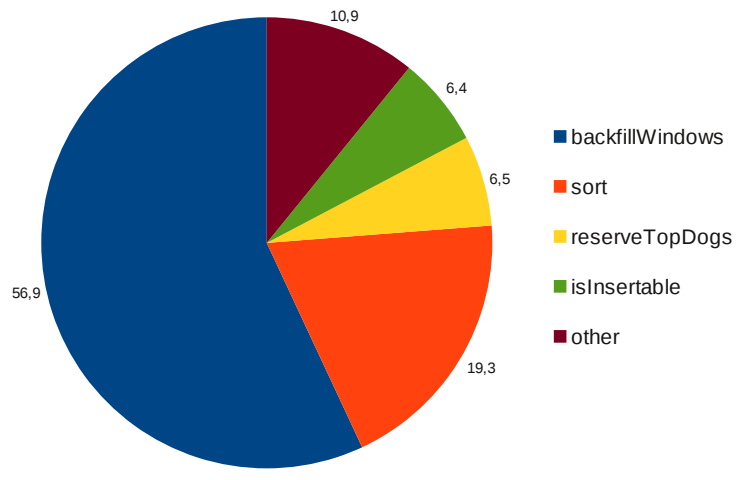

\section{Results}

- Bigger part for backfill windows

- But efficient job placement

- $20 \%$ for sorting 


\section{Ideas for parallelization}

- I/O: Parallel parsing of job and node data, parallel output

- Job sorting: job priorities are calculated independently $\rightarrow$ parallel sorting

- Scheduling algorithms: parallel search for suitable jobs at each time step

- Resource manager: parallel search for suitable compute nodes, torus search 